
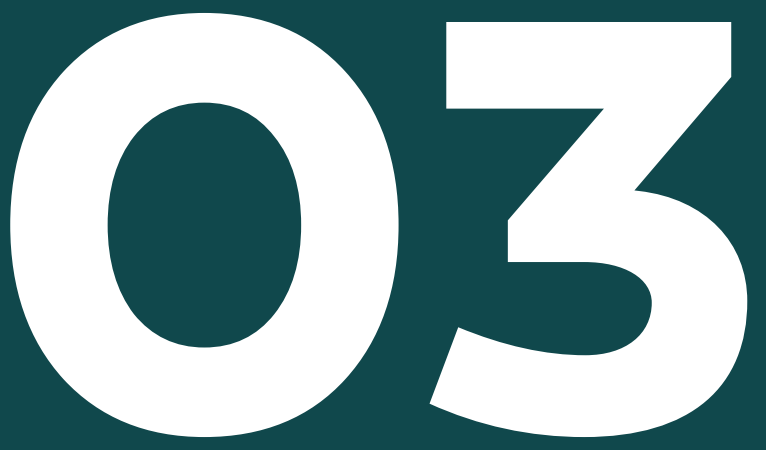

\title{
El Benchmarking como herramienta para el logro de calidad y eficiencia ante la burocratización mecanizada en los servicios de las instituciones
}

Benchmarking as a tool to achieve quality and efficiency in the face of mechanized bureaucratization in the services of the institutions

FECHA DE RECEPCIÓN: 31/01/2021

FECHA DE APROBACIÓN: 07/07/2021

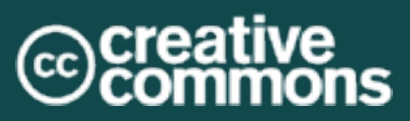




\section{Resumen}

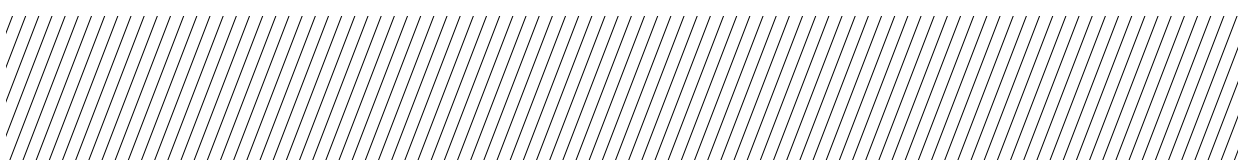

\section{Pamela del Rocío Fajardo Vanegas ${ }^{1}$ Hugo Daniel Cabrera Vinueza ${ }^{2}$ María Esther Alcántara Gutiérrez ${ }^{3}$ Armando Adrián Millán Dumas ${ }^{4}$}

Los servicios prestados por las organizaciones son evaluados por los usuarios en sus procesos, el estilo de atención y la satisfacción que brindan sus resultados. Esta investigación analizó la experiencia que los usuarios han tenido con la atención que reciben en los servicios públicos, formulando una crítica constructiva a la burocratización mecanizada. El principal objetivo de la investigación fue determinar cómo afectan los procesos burocratizados a los usuarios del servicio en tiempo, costo y beneficio. La metodología seguida fue un análisis crítico deductivo correlacional, con un planteamiento heurístico, como base de cambio y solución a los problemas de la burocratización en los procesos. Esta investigación analizó la experiencia que los usuarios han tenido con la atención que reciben en los servicios públicos, a fin de identificar los indicadores para una propuesta de mejora, que contrarreste la burocratización mecanizada mediante la aplicación de la herramienta de Benchmarking. Se buscó alternativas de cambio en la gestión administrativa, ya que bien fundamentada es un conductor del mejoramiento en los trámites y de la satisfacción del usuario. Los resultados mostraron que la insatisfacción es moderadamente alta y que las instituciones públicas, en comparación con las privadas, necesitan reestructura e innovación inmediata.

Palabras clave: benchmarking, burocratización mecanizada, calidad de servicio, procesos, satisfacción del cliente.

\footnotetext{
1 Instituto Superior Tecnológico Japón ·pfajardo@itsjapon.edu.ec • Quito, Ecuador

2 Instituto Superior Tecnológico Japón · hcabrera@itsjapon.edu.ec · Quito, Ecuador

3 Universidad Benito Juárez-Puebla · dir.academica@ubjonline.mx · Puebla, México

4 Universidad Benito Juárez-Puebla · amdasesorubj@gmail.com · Puebla, México
} 


\section{Abstract}

T

he services provided by the organizations are evaluated by the users in their processes, the style of attention, and the satisfaction that their results provide. This research analyzed the experience that users have had with the care they receive in public services, thus formulating a constructive criticism of mechanized bureaucratization. The main objective of the research was to determine how to control the bureaucratized processes to the users of the service in time, cost and benefit The methodology followed was a correlational deductive critical analysis, with a heuristic approach as a basis for change and solution to the problems of bureaucratization in processes. This research analyzed the experience that users have had with the care they receive in public services to identify the indicators for a proposal for improvement, which counteracts mechanized bureaucratization through the application of the Benchmarking tool, to find alternatives for change in the administrative management, since well-founded is a driver of improvement in procedures and user satisfaction. The results obtained show that dissatisfaction is moderately high, and that public institutions compared to private ones need immediate restructuring and innovation.

Keywords: Benchmarking, mechanized bureaucratization, processes quality of service, customer support.

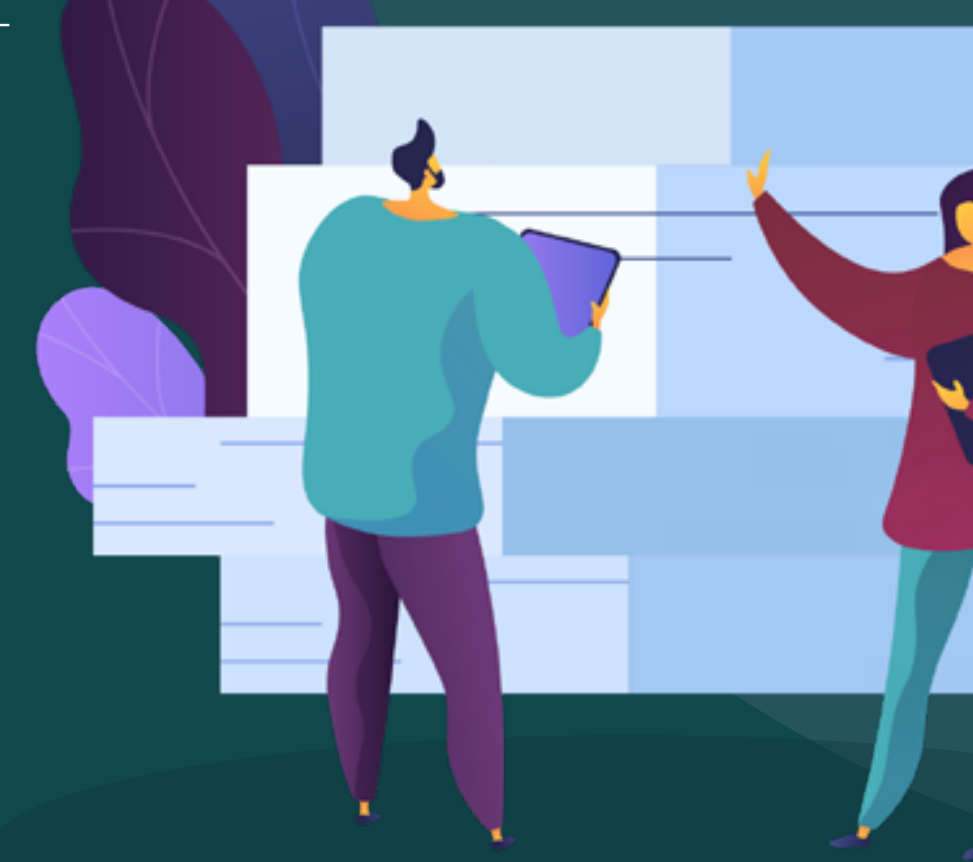


Un estudio realizado por Bozeman (2015), demostró los niveles de burocratización de los países con relación al empleo público, determinó que el total de empleados que trabajan en un gobierno van desde el $5 \%$ en Corea hasta cerca del 30\% Noruega, sin contar el aumento excesivo en América Latina (Bozeman, 2015). Rodríguez (1987), en su escrito, definió que no se puede cuantificar en su totalidad la burocratización debido a que cada organización estatal tiene una problemática de burocracia diferente o contiene un sinnúmero de problemas administrativos sin resolver, los cuales, se han ido derivando de otras administraciones que no han buscado solución, generando el efecto de bola de nieve; por lo tanto, se puede mencionar que la burocratización se impone por la estructura funcional y jerárquica de una organización. Además, se dice que las instituciones con este tipo de sistema deben aprender a delegar y a dividir el trabajo de forma similar en todos sus departamentos, con el fin de lograr el desarrollo adecuado de los procesos sin retrasos y sin demoras innecesarias (Bellidov, 2017).

La burocratización es más evidente en el sector público, porque el control no abarca a todas las acciones que se realizan de manera interna. Ello, sumado a que las autoridades competentes son temporales, por lo tanto, sus objetivos no están alineados a resultados $y$, muchas veces, quien trabaja es parte de una concesión política, como una prebenda ganada, por lo que se llega a presentar ineficiencia o corrupción. En Ecuador hay evidencias y estudios que demuestran que las instituciones públicas presentan burocratización en sus administraciones, lo cual, le lleva a la ineficiencia desde hace algunas décadas.

En una investigación realizada en el 2015 al Servicio de Rentas Internas (SRI), institución gubernamental de Ecuador, se encontró que hay un amplio consenso, tanto público como académico, con referencia a la 'burocratización'; es decir, en este tipo de entidades estatales centra en su administración, el ideario burocrático como cultura organizacional, estableciendo a la institución, la connotación peyorativa que implica falta de eficacia y eficiencia en sus actividades, normas y procedimientos (Dávila Espinosa, 2015).

Otro estudio del mismo año (2015), realizado por el Instituto Nacional de Estadísticas y Censos (INEC) sostiene que, a decir de las personas, las instituciones públicas se mantienen igual en los siguientes aspectos: En los servicios que brindan a los ciudadanos con un $62,8 \%$, en 
la información que recibe el ciudadano en un $62,6 \%$, en instalaciones de oficinas de atención al público con el $59,1 \%$, en profesionalidad del personal con un $62,9 \%$ y en procesamiento de quejas y sugerencias de los ciudadanos con un $70 \%$. Es decir, las instituciones públicas del Ecuador no han mejorado sus procesos burocráticos ni de atención al cliente, así se incorporen prácticas de cambio organizacional y social (INEC, 2015).

Desde otra perspectiva y tomando como referencia la encuesta realizada por el INEC en el año 2008, se estableció que el 14,3\% de quejas y reclamos en las instituciones públicas es por falta de información, el $15,8 \%$ por falta de profesionalismo de los funcionarios y $24,6 \%$ porque el personal de ventanilla no comprendió el problema del ciudadano (Zapata Mora, 2018). Ecuador, en los últimos años, según el informe del Banco Interamericano de Desarrollo (BID) denominado Diagnóstico Institucional del Servicio Público de Ecuador, se convirtió en el sexto país con altos índices de ineficiencia en servicio público luego de evaluar a otros 15 países del continente americano (Zapata Mora, 2018).

La burocratización afecta los resultados administrativos de las organizaciones e impacta, volviendo deficiente la calidad de atención al usuario al no considerar la satisfacción del cliente como eje de todas las iniciativas de mejora. Esto hace a las instituciones perder clientes que no desean regresar a una organización donde el reproceso y la demora es el factor diario; en tanto que las instituciones públicas pierden su reputación social, lo que repercute de manera política, económica y social, a largo plazo.

Dentro de la administración privada, la burocratización, en cambio, se le denomina falta de 'cultura de servicio al cliente', porque hace referencia a las metodologías que las personas deben aprender para organizar la realidad institucional ante el cliente final.

En el ámbito privado, tiene mayor observación y condición la satisfacción de los clientes finales. El autor Rigoberto E. Gómez Cárdenas (2016) en su artículo: La Calidad en los Servicios desde la Perspectiva del Consumidor: El caso de EPMAPA-SD, aplicando el modelo SERVQUAL, determinó que la insatisfacción nunca superó la mitad más uno; lo más alto de la insatisfacción de los clientes llegó al 49,1\%, en algunas dimensiones del modelo aplicado (Gómez Cárdenas, 2016). 
Pérez (2014) afirmó que "toda acción en la prestación del servicio debe estar dirigida a lograr la satisfacción en el cliente". Sin olvidar que la calidad es importante, pero, la entrega y el amor al cliente hacen que las actividades empresariales sean las herramientas que contribuyan alcanzar el éxito deseado a lo largo del tiempo (Pérez , 2014).

Desde este punto de vista, se puede resaltar que el servicio al cliente es la clave primordial para el éxito de cualquier tipo de organización y, más aún, las que prestan servicio; recordar que para dar un buen servicio al cliente hay que crear primero un vínculo directo con él y así lograr una relación duradera, creando confianza, empatía, etc.; hay que ponerse en su lugar y comprender sus necesidades, de modo que el cliente se sienta amado y comprendido (Gutiérrez Cadena, 2019).

La calidad de servicio representa una de las variables consideradas clave por los expertos para la competitividad de una organización, más aún, si quieren permanecer en un mercado competitivo donde los clientes, más que un producto, buscan experiencias; por tanto, la calidad del servicio se convierte en un elemento estratégico que confiere ventaja diferenciadora (Mendoza Aquino, 2009).

El tener presente a la calidad como el factor principal de cambio y éxito organizacional, conlleva al mejor desempeño del capital humano que opera en las áreas estratégicas y administrativas, sin importar la naturaleza de las instituciones. 


\section{Metodología}

Se aplicó el método deductivo - heurístico - correlacional, iniciando con una indagación exhaustiva de lo teórico-crítico de todos aquellos autores que abordan los temas correlacionados con la burocracia, el burocratismo, la calidad del servicio y la atención al cliente, así como la satisfacción cuando el proceso se realiza con calidad y calidez; además, el impacto que genera en la reputación de una organización, ya sea pública o privada y sus efectos a largo plazo, con el fin de determinar cómo afectan los procesos burocráticos en tiempo-costo-beneficio ante el grado de satisfacción del cliente. Adicionalmente, se aplicó un corte transversal con enfoque cuantitativo, mediante un instrumento creado exprofeso para la medición de la calidad y la satisfacción/insatisfacción por los servicios recibidos en las instituciones.

La herramienta de investigación que facilitó la identificación y la determinación de las causas y efectos en cada una de las variables es la Matriz de Vester, una técnica de descomposición lógica de las relaciones que causan consecuencias en la satisfacción de los usuarios, permitiendo llegar a los orígenes básicos del porqué se genera la burocratización en los procesos internos de las instituciones. Posteriormente, se ejecutó una exploración sobre cómo la herramienta de Benchmarking puede contribuir a la construcción de la solución para flexibilizar los procesos burocráticos en las instituciones.

El cuestionario para la recolección de datos fue de autoría propia, estructurado en una serie de quince (15) preguntas organizadas en cuatro (4) bloques temáticos mixtos:

Bloque de Información general: analizar perfiles de la muestra;.

Bloque de Atención al cliente: percepción emocional y experiencias de los clientes

Bloque de Satisfacción al cliente: atención al cliente, manejo de las quejas e insatisfacción.

Bloque de Imagen institucional: valor institucional. 
El instrumento presentó el esquema esperado porque se le aplicó el método Delphi, que consintió en quitar la ambigüedad y sumarle rigor metodológico, así como también el índice de concordancia de Kappa de Fleiss, que se considera aceptable, cuando es mayor a 0,61, lo cual, representa una fuerza de concordancia entre los peritos que participaron en los dos pilotos, para definir si el instrumento de investigación es bueno o muy bueno (Torres Gordillo \& Perera Rodriguez, 2009).

Se desarrollaron dos rondas de evaluación del instrumento; la primera, con diez (10) profesionales de distintas áreas para establecer ítems de claridad, coherencia, suficiencia, relevancia y esencia, las recomendaciones se plasmaron en los ajustes necesarios con el fin de determinar el punto de viaje entre la experiencia del usuario y las emociones que genera la satisfacción/insatisfacción. La segunda evaluación fue un sondeo con veinticinco (25) personas que permitió examinar el nivel metodológico.

El modelo de muestreo que se aplicó en este estudio es el no probabilístico (Hernández Sampieri \& Mendoza Torres, 2018) por conveniencia, que también se le denomina 'muestras dirigidas', debido a que la selección de los individuos fue a través de los usuarios que tenían disponibilidad para formar parte de la muestra en el tiempo determinado que se llevó a cabo el estudio.

El tipo de muestreo se desarrolló durante la pandemia del Covid-19, en el año 2020, el cual, limitó el obtener la información de toda la población requerida, siendo esta la razón de seleccionar a los miembros efectivos para hacer que la muestra sea representativa, buscando obtener las opiniones y puntos de vista sobre la calidad de servicio que brindan las instituciones públicas y privadas a los usuarios; lo más importante, fue que la representatividad la determinó el equipo investigador, puesto que, se abrió a la voluntad de las personas que desearon ser parte de la muestra y brindaron la accesibilidad al momento de llenar el instrumento digital de medición.

De este modo, la estimación de la muestra para este estudio con población infinita se calculó basada en los siguientes parámetros: Nivel de Confianza al $95 \%$, es decir $Z=1.96$, valor de probabilidad de éxito, igual a p0.5, valor de fracaso igual a q0.5 y el valor del error máximo de $e=5 \%$ o (0.05); y, después de la aplicación de la respectiva fórmula se 
pudo establecer que el tamaño de la muestra fue de 384 personas, correspondiendo al número de individuos encuestados que voluntariamente llenaron el cuestionario de forma digital, por medio de la herramienta de Google formularios (Google Forms). Por último, la aplicación del cuestionario se realizó en los meses de julio a septiembre del año 2020.

\section{Resultados}

En este apartado, se presentan los resultados obtenidos en el proceso de investigación:

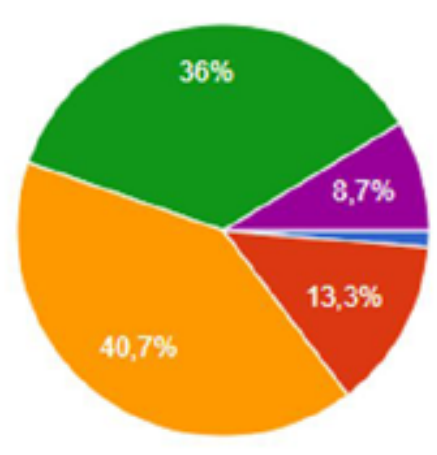

Figura 1: Resultados sobre atención al cliente en instituciones públicas. Elaborado por: Los investigadores.

El $40,7 \%$ de los encuestados mencionan que la atención en las instituciones públicas no es ni buena ni mala, teniendo una actitud imparcial en relación a la experiencia vivida al momento de realizar alguna gestión administrativa; el 36\% aludió que la atención diariamente es mala ya que no velan por el bienestar de los usuarios ni por la calidad en los servicios. Por otro lado, un 13,3\% expresó que es buena, así como el $1,3 \%$ que es muy buena porque se han realizado cambios significativos demostrando avances positivos en la atención; pero, hay un $8,7 \%$ que discrepa totalmente mencionando que la atención es muy mala porque 
no hay sentido de pertenencia por la institución ni el valor hacia el usuario final.

Más del $50 \%$ de los encuestados que respondieron los factores cualitativos, apuntaron que las instituciones públicas fallan en su atención al usuario porque:

- Tienen ineficiencia en los procesos y trato desfavorable al cliente.

- Existe mucha burocracia y papeleo en ventanilla.

- Tienen procesos lentos y retrógrados, y,

- Existe poco personal y muchas tareas para cada servidor.

Como se puede apreciar, cada aspecto hace referencia a opiniones que fueron señalados por los encuestados, ya que ellos percibieron desagrado por la atención al cliente en las instituciones públicas, haciendo notorio que el gran papeleo que causa la burocratización afecta a procesos internos y a la calidad de atención que se debe dar a un usuario final.

A diferencia de lo que ocurre en una institución privada, las respuestas ponen en contexto los resultados que difieren en la percepción del usuario, lo cual, llama la atención, pues, el contraste resulta revelador sobre la actitud que una y otra organización sigue y que es percibido con claridad por el usuario en función de la atención por el servicio recibido. 


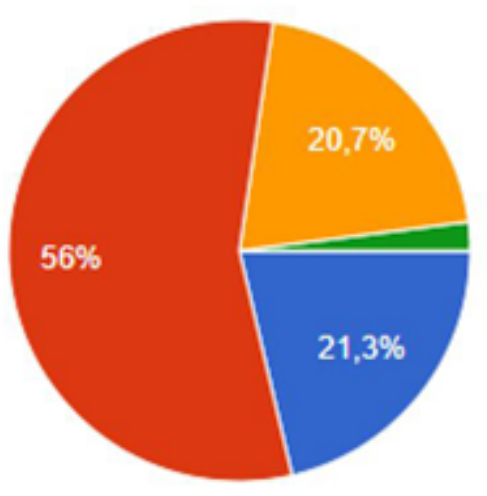

Muy Buena

Buena

Ni Buena ni mala

- Mala

Muy mala

Figura 2: Resultados sobre atención al cliente en instituciones privadas Elaborado por: Los investigadores.

Sobre la atención al cliente en las instituciones privadas, un 56\% de los encuestados mencionó que es buena, debido a los procesos y a la calidad que brindan a los usuarios; seguido del 21,3\% que señaló que es muy buena, ya que existen soluciones oportunas a los problemas de los usuarios, principalmente, aplicando el factor de eficacia. También, un $20,7 \%$ se mantuvo imparciales definiendo que la atención para ellos no es ni buena ni mala, porque no han recibido lo que lo esperaban; por último, una diferencia del $2 \%$ han dicho que es igual de mala que la pública, tienen procesos, pero, solo piensan en el dinero y no en el bienestar del usuario.

Los aspectos más comentados en las preguntas cualitativas que generan excelencia en la atención al cliente en las instituciones privadas fueron:

- Existe planificación y concordancia con su filosofía organizacional.

- Existen procesos de seguimiento y evaluaciones estrictas. 
- El cliente es importante y valoran su tiempo.

- Los empleados dan lo mejor de ellos y quieren conservar su trabajo.

Los ítems que fueron mencionados con anterioridad son las características señaladas por los encuestados, demostrando una divergencia, porque en lo privado el usuario es el motor de la institución; sin clientes no hay ingresos y además porque se usa como diferenciador de productos o servicios para ganar mercado. En consecuencia, los resultados en las respuestas son sumamente consistentes, lo que hace pensar que va más allá de una imagen subjetiva, sino que se coloca en el horizonte de lo real.

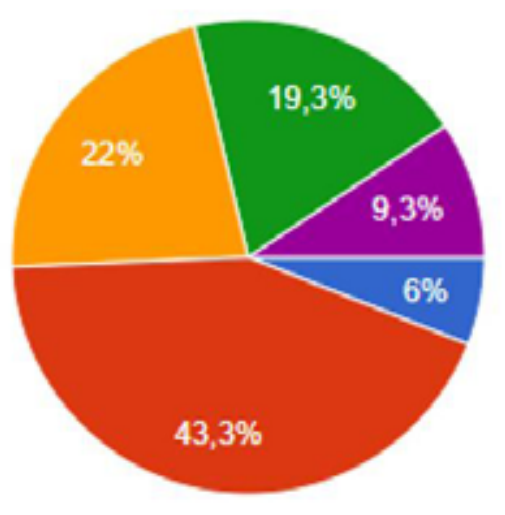

Muy satisfecho

Poco satisfecho

Indiferente

Insatisfecho

Nada satisfecho

Figura 3: Resultados de la atención al cliente recibido en las instituciones públicas

Elaborado por: Los investigadores. 
En el rendimiento de la atención al cliente en las instituciones públicas, un 43,3\% mencionó que están un poco satisfechos con la atención porque, dependiendo del lugar y de la institución pública, han tenido resultados a sus solicitudes, pero, que los procesos son escasos y confusos, ya que no orientan y generan pérdida de tiempo; asimismo, que el existir mucha 'tramitología' (burocratización) hacen que el usuario tenga que visitar muchos departamentos con pocas soluciones efectivas.

En segundo plano, el 22\% de los encuestados se sigue manteniendo indiferente ante las respuestas sobre la satisfacción, ya que no han logrado cubrir al 100\% las necesidades, pero, después de una larga espera, han logrado obtener el trámite que era el objetivo central de su visita.

Asimismo, el 19,3\% se han sentido insatisfechos, porque la atención no ha cumplido con sus expectativas. El factor tiempo juega el papel más importante, ya que han tenido que esperar mucho para recibir el servicio, además el personal no ha brindado la calidad adecuada al usuario, evidenciando desorden y mal humor.

Para finalizar, el 9,3\% se siente nada satisfecho con el servicio, principalmente, porque nunca recibieron una solución a su problema; al contrario, comprobaron que la atención es lenta y con muchos 'padrinazgos' por la corrupción, para obtener resultados. Mientras tanto, el 6\% restante determinó que están muy satisfechos con la atención al usuario en el sector público, ya que sí pudieron obtener su trámite en el tiempo que lo proyectaron.

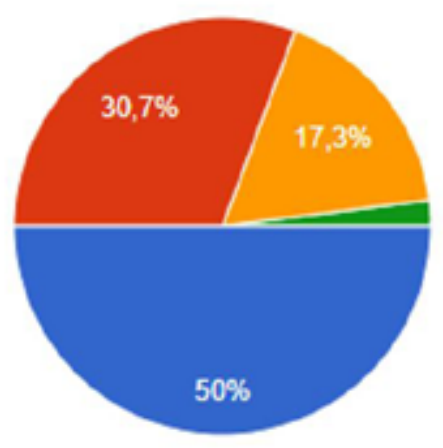

Muy satisfecho

Poco satisfecho

Indiferente

Insatisfecho

Nada satisfecho

Figura 4: Resultados de la atención al cliente recibida en las instituciones privadas

Elaborado por: Los investigadores. 
Respecto a la 'atención al cliente' en las instituciones privadas se ha podido establecer que el $50 \%$ de los encuestados están muy satisfechos, ya que estas instituciones exteriorizan calidad, bienestar, vanguardia y calidez hacia el usuario final; por tanto, el personal interno siempre brinda una buena atención porque están capacitados, bien informados, ayudando a solucionar problemas y siendo el beneficiario el protagonista del proceso, de acuerdo con la literatura especializada, como elementos distintivos del buen servicio.

De tal manera que, el 30,7\% insinuó que también están un poco satisfechos ya que si alguna vez tuvieron una mala experiencia, dependió de la institución o de la persona que brindó el servicio y no cumplió con la normativa interna. Por otra parte, el 17,3\% son indiferentes puesto que han experimentado que la atención sí es mejor que el sector público, pero, todavía tienen personal que no está dispuesto a colaborar con los usuarios o que el sentimiento de servicio no es prioridad.

El 2\% sobrante se sienten insatisfechos porque hay instituciones que no generan sus know-how de forma rápida y precisa, fundando decepción en los beneficiarios que no han podido resolver sus dudas o han tenido que reprocesar una y otra vez el trámite hasta encontrar la solución. Entre los procesos comunes que prestan las instituciones públicas, justamente, está la demanda de servicios relacionados con los trámites relacionados a lo largo de la vida del ciudadano, y sus funciones básicas para la vida y para vivir en sociedad.

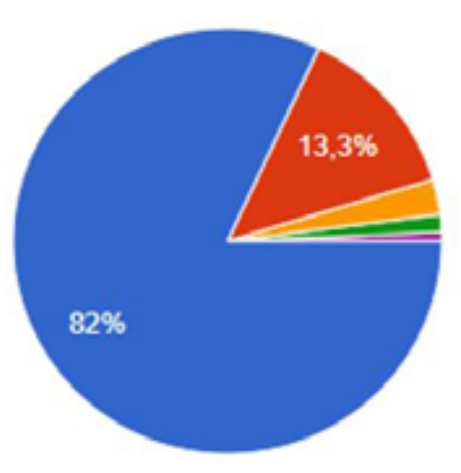

Muy de acuerdo

De acuerdo

Ni de acuerdo ni en desacuerdo

En desacuerdo

Muy en desacuerdo

Figura 5: Resultados sobre el manejo de procesos correctos en instituciones públicas

Elaborado por: Los investigadores. 
Al encuestar sobre la importancia de la agilidad que deben tener los procesos en las instituciones públicas, el $82 \%$ de la muestra están muy de acuerdo con que la atención al usuario en el sector público es un elemento importante; así también, un 13,3\% están de acuerdo que lo procesos deben ser muy ágiles y estables para brindar una excelente atención. Por otro lado, el 4,7\% de la muestra se distribuyó entre los otros factores, siendo la minoría de los usuarios; con esto queda demostrado que el tener procesos claros y ágiles es el diferenciador que las instituciones públicas necesitan para los cambios en la atención al ciudadano.

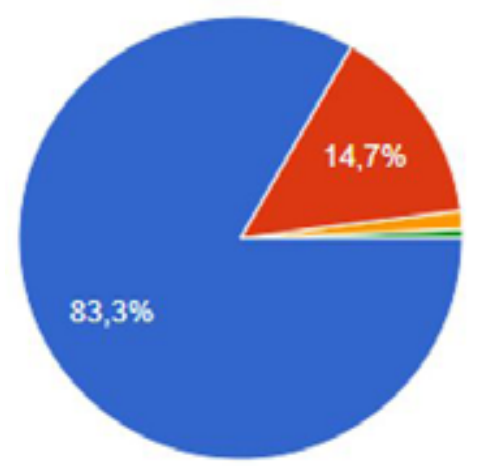

Muy de acuerdo

De acuerdo

Ni de acuerdo ni en desacuerdo

En desacuerdo

Muy en desacuerdo

Figura 6: Resultados sobre el manejo de procesos correctos en instituciones privadas

Elaborado por: Los investigadores.

En cuanto a la importancia de la agilidad que deben tener los procesos en las instituciones privadas, un $83,3 \%$ de personas están muy de acuerdo que la atención al usuario es lo que más hace la diferencia en el sector privado; así también, un 14,7\% están de acuerdo que lo procesos son ágiles y estandarizados cuando existe una excelente gestión administrativa. De tal forma que, tan solo el $2 \%$ de quienes respondieron, se inclinan por otros factores de la pregunta, demostrando así, que para los beneficiarios, siempre lo más importante será la eficiencia en los procesos y esto se da cuando las actividades y los recursos están bien gestionados haciendo que no exista burocratización. 

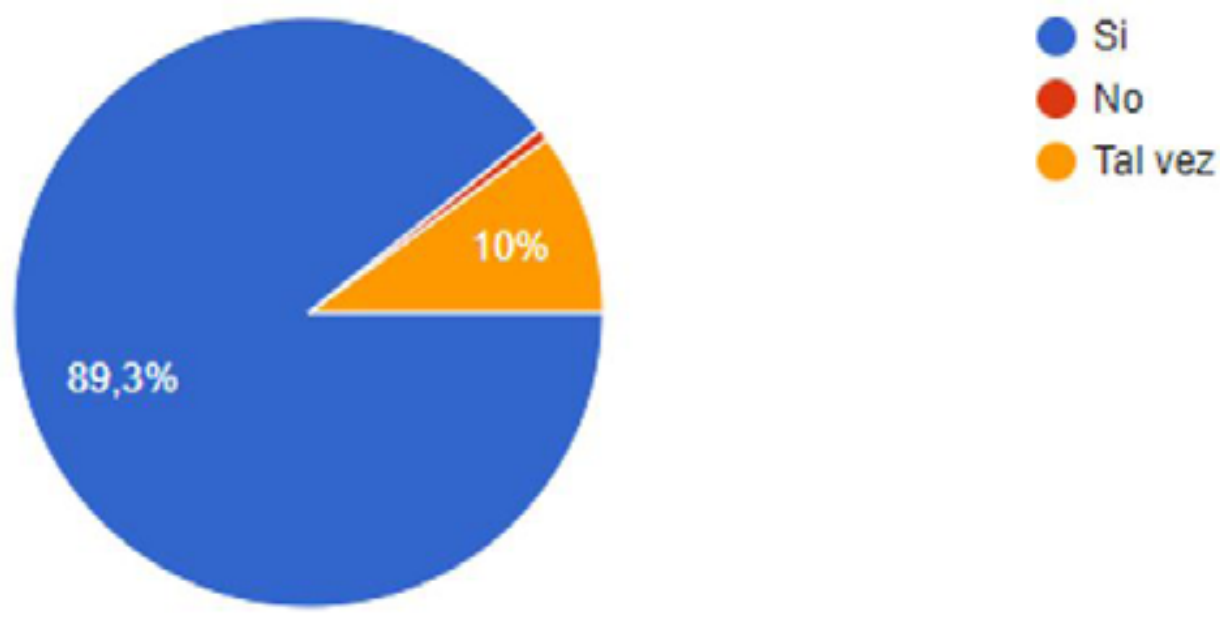

Figura 7: Resultados sobre disminuir la burocratización en las instituciones públicas

Elaborado por: Los investigadores.

El propósito también fue observar las diferencias significativas en las respuestas del grupo evaluado; con la intención de conocer sus opiniones se incluyó una valoración importante que contenga dos tipos de respuestas, una cualitativa y una cuantitativa, estableciendo que si se disminuye la burocratización en los procesos institucionales se puede garantizar calidad en la atención al usuario en los servicios administrativos.

Los resultados que presentó esta pregunta fueron: el 89,3\% dijeron que sí es importante disminuir la burocratización en los procesos porque, mientras más rápido se pueda dar solución a un trámite, es mucho mejor para el usuario, ya que esto da una excelente imagen de la institución y no se pierde el tiempo productivo; así también, porque muchos procesos y trámites no quedarían estancados y el usuario quedará totalmente satisfecho.

Así mismo, un 10\% mencionó que tal vez si disminuye la burocratización en los procesos puede mejorar el servicio, pero, esto no garantiza una calidad de atención al usuario en un cien por ciento, puesto que la atención se puede perfeccionar si existiera mayor tecnología, mejor comunicación con accesos virtuales que admitan a los usuarios a conocer 
requisitos y normativas vigentes y sin tanta confusión con el papeleo por cada trámite; además, con la digitalización se lograría un alto grado de satisfacción y confianza de los usuarios, porque se vería un ahorro de tiempo, dinero y se evitaría el desgaste público en entidades que no generan ningún valor al Estado.

Los factores cualitativos que más se destacaron en las respuestas de los encuestados fueron:

- Falta de reglamentación interna haciendo los procesos muy extensos.

- Alto tiempo de espera en la atención al usuario.

- Incorrecto manejo de la información que se brinda al usuario en ventanilla.

- Falta de cultura de servicio al usuario.

- Falta de motivación y capacitación del personal interno.

- Incumplimiento en plazos de entrega a los trámites del usuario.

- Deficiente planificación administrativa al interior de las instituciones.

- Falta de cobertura y automatización de los procedimientos. 


\section{Discusión}

Schiffman \& Lazar Kanuk (2011), establecieron en su libro que la relación que se presenta entre la satisfacción y la ansiedad de los usuarios con el tiempo de espera se ve reflejado en las actitudes y en el estados de ánimo; es decir, los usuarios menos satisfechos no se sienten bien con la atención que reciben de las instituciones, principalmente, las públicas, cosa se hace reflexionar sobre las fallas y la burocratización que presentan este tipo de instituciones al realizar un trámite administrativo. Además, se pudo detectar que la falta de espacios de orientación al ciudadano hace que no se permita las labores técnicas adecuadas para la satisfacción de las necesidades del usuario. A esto se suma la escasez de recursos tecnológicos junto a la poca experiencia del personal, fomentando así a que los procesos sean burocráticos, perdiendo la confianza de los usuarios porque no existe una inversión en la calidad organizacional y en la aplicación de una buena cultura de servicio al consumidor.

En definitiva, todas las respuestas cualitativas aportadas por los encuestados sirvieron como lluvia de ideas, para elaborar un análisis más específico a través de la Matriz de Vester, que consiente en identificar parámetros con las debilidades institucionales y los hechos o circunstancias que la rodean y le afectan para el cambio esperado.

El proceso de elaboración de la matriz es:

- Conformar una lista con los problemas seleccionados de la lluvia de ideas.

- Construir la matriz calculando el grado de influencia de cada uno de los problemas en las filas, anotando la calificación en la columna respectiva a cada ítem, de acuerdo con la siguiente valoración con base al grado de causalidad:

- No la causa: (0)

- Causalidad muy débil: (1) 
- Causa semidirecta: (2)

- Causa directamente fuerte: (3) (Pérez Leal, 2018).

A continuación, se presentan, bajo un análisis, los resultados obtenidos entre los factores y la problemática central, para la detección de las complicaciones críticas en este estudio.

Tabla 1: Matriz de Vester con la situación problemática de la burocratización en las instituciones públicas generando insatisfacción en los usuarios.

\begin{tabular}{|c|c|c|c|c|c|c|c|c|c|c|c|}
\hline $\begin{array}{l}\text { CÓDI- } \\
\text { GO }\end{array}$ & VARIABLE & P1 & P2 & P3 & P4 & P5 & P6 & P7 & P8 & $\begin{array}{l}\text { INFLU- } \\
\text { ENCIA }\end{array}$ & \\
\hline P1 & $\begin{array}{l}\text { Falta de reglamentación } \\
\text { interna haciendo los pro- } \\
\text { cesos burocráticos. }\end{array}$ & 0 & 0 & 3 & 2 & 1 & 0 & 2 & 0 & 8 & \\
\hline P2 & $\begin{array}{l}\text { No hay una valoración } \\
\text { costo-beneficio de la de- } \\
\text { ficiencia. }\end{array}$ & 3 & 0 & 3 & 3 & 3 & 3 & 3 & 3 & 21 & 11 \\
\hline P3 & $\begin{array}{l}\text { Falta de cultura de servi- } \\
\text { cio al usuario. }\end{array}$ & 2 & 2 & 0 & 1 & 2 & 1 & 2 & 1 & 11 & \\
\hline P4 & $\begin{array}{l}\text { Falta de capacitación } \\
\text { pertinente del personal } \\
\text { interno. }\end{array}$ & 0 & 2 & 3 & 0 & 3 & 2 & 3 & 0 & 13 & \\
\hline P5 & $\begin{array}{l}\text { Falta de motivación al } \\
\text { personal interno. }\end{array}$ & 1 & 2 & 3 & 2 & 0 & 2 & 3 & 0 & 13 & \\
\hline P6 & $\begin{array}{l}\text { Falta de desarrollo orga- } \\
\text { nizacional e imagen cor- } \\
\text { porativa. }\end{array}$ & 2 & 3 & 3 & 3 & 3 & 0 & 3 & 2 & 19 & \\
\hline P7 & $\begin{array}{l}\text { Falta de identificación } \\
\text { de trazabilidad, tiempos } \\
\text { logística e información en } \\
\text { procesos y trámites. }\end{array}$ & 3 & 3 & 3 & 2 & 1 & 2 & 0 & 3 & 17 & \\
\hline \multirow[t]{2}{*}{ P8 } & $\begin{array}{l}\text { Falta de automatización } \\
\text { de los procedimientos. }\end{array}$ & 1 & 2 & 1 & 2 & 1 & 3 & 1 & 0 & 11 & \\
\hline & & P1 & P2 & P3 & P4 & P5 & P6 & P7 & P8 & & \\
\hline eje y & \multirow[t]{2}{*}{ DEPENDENCIA } & 12 & 14 & 19 & 15 & 14 & 13 & 17 & 9 & & \\
\hline & & & & 8 & & & & & & & \\
\hline
\end{tabular}

Elaborado por: Los investigadores. 
El resultado obtenido en la matriz, luego de la valoración casual, dio que la influencia con más valor obtenido fue: no hay una valoración costo-beneficio de la deficiencia, con veintiuno (21) puntos, siendo este el problema que deriva a los demás de la tabla 4. La segunda influencia que demostró valores comparativamente altos fue: la falta de desarrollo organizacional e imagen corporativa, con un valor de diecinueve (19) puntos.

Los problemas ponderados en el eje de la dependencia coinciden también con la influencia, donde P3 posee el mayor valor con diecinueve (19) puntos, seguida de P7 con diecisiete (17) puntos; esto demuestra que la falta de cultura de servicio al usuario y la falta de identificación de trazabilidad, tiempos logística e información en procesos y trámites, que constituyen los problemas con mayor énfasis en este tipo de instituciones, lo cual, se ve reflejado en la atención que brindan al ciudadano.

En el caso algunos de los problemas identificados se pueden solucionar con estrategias de capacitación al personal que viven de cerca el proceso de atención al usuario, aunque esto no eliminaría del todo la insatisfacción del ciudadano, pero, podría contribuir a que el personal interno comprenda y cubra las necesidades que el usuario está buscando satisfacer.

Mientras tanto, los criterios que están relacionados, por ejemplo, con la naturaleza crítica de un proceso y las prácticas asociadas a la satisfacción del usuario final, siempre van de la mano con la importancia que el cliente debe tener en la cadena interna de la organización; es decir, la satisfacción de los usuarios finales van relacionados directamente con la importancia de los planes de acción y de las estrategias de mejora que las instituciones deben plantear para resolver problemas de sus beneficiarios.

Luego de este proceso se sumaron los valores resultantes de las columnas y las filas, los cuales, son presentados a través de un gráfico de coordenadas. Según su ubicación, los problemas se clasifican en: críticos, activos, pasivos o indiferentes. 


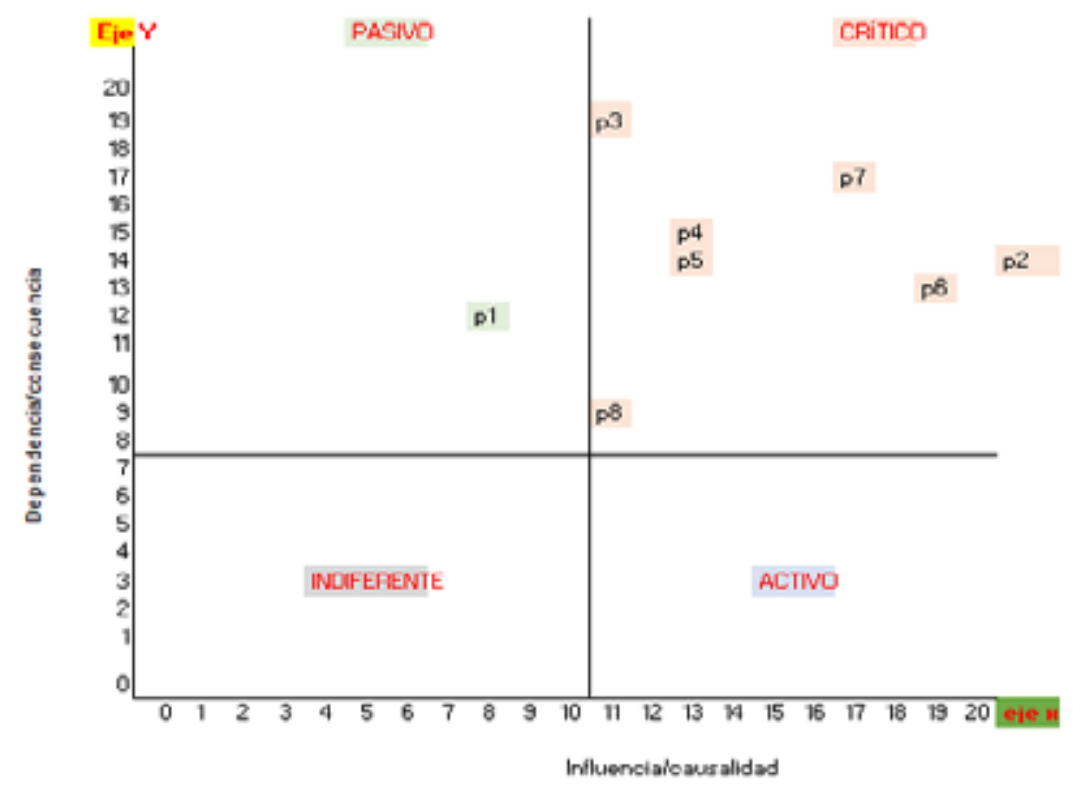

Figura 8: Configuración de la problemática central

Elaborado por: Los investigadores.

En el análisis de los cuadrantes, lo que se buscó es examinar los principales problemas que existen en las instituciones públicas y así determinar el nivel con mayor relevancia, con el fin de establecer los factores que causan mayores dificultades en la gestión administrativa, para generar estrategias y recomendaciones que sirvan a otros estudios posteriores con base a los hallazgos arrojados en este análisis de Vester. Lo encontrado se presenta a continuación:

Cuadrante pasivo: Se localiza el factor P1, el cual, se concibe como un problema sin gran influencia causal sobre los demás, pero, se originó por la mayoría, siendo la falta de reglamentación uno de los elementos que mantiene la burocratización.

Cuadrante crítico: Se evidencian los factores P2, P3, P4, P5, P6, P7 y P8, los cuales, se entienden como problemas de gran causalidad, que a su vez son originados por la mayoría de los problemas existentes. Requieren de gran cuidado en su análisis, ya que de sus intervenciones, dependen los resultados finales. 
Por ende, en las instituciones públicas al no tener una valoración costo-beneficio por el usuario, se presenta la falta de cultura de servicio, ya que no hay desarrollo organizacional e imagen corporativa, dando como consecuencia la falta de motivación del personal, la ineficiencia en la trazabilidad, en los tiempos de la logística y en la información de los procesos, porque no son automatizados y, también, porque no hay capacitación permanente al personal interno. Asimismo, por medio del análisis de resultados y la óptica del marco teórico se confirmó la hipótesis planteada y se afirmó que aplicar una herramienta de Benchmarking al interior de las instituciones públicas, efectúa el cambio en los procesos y en la cultura de servicio al cliente.

Tabla 2: Aplicación de Benchmarking para mejorar los resultados en el servicio

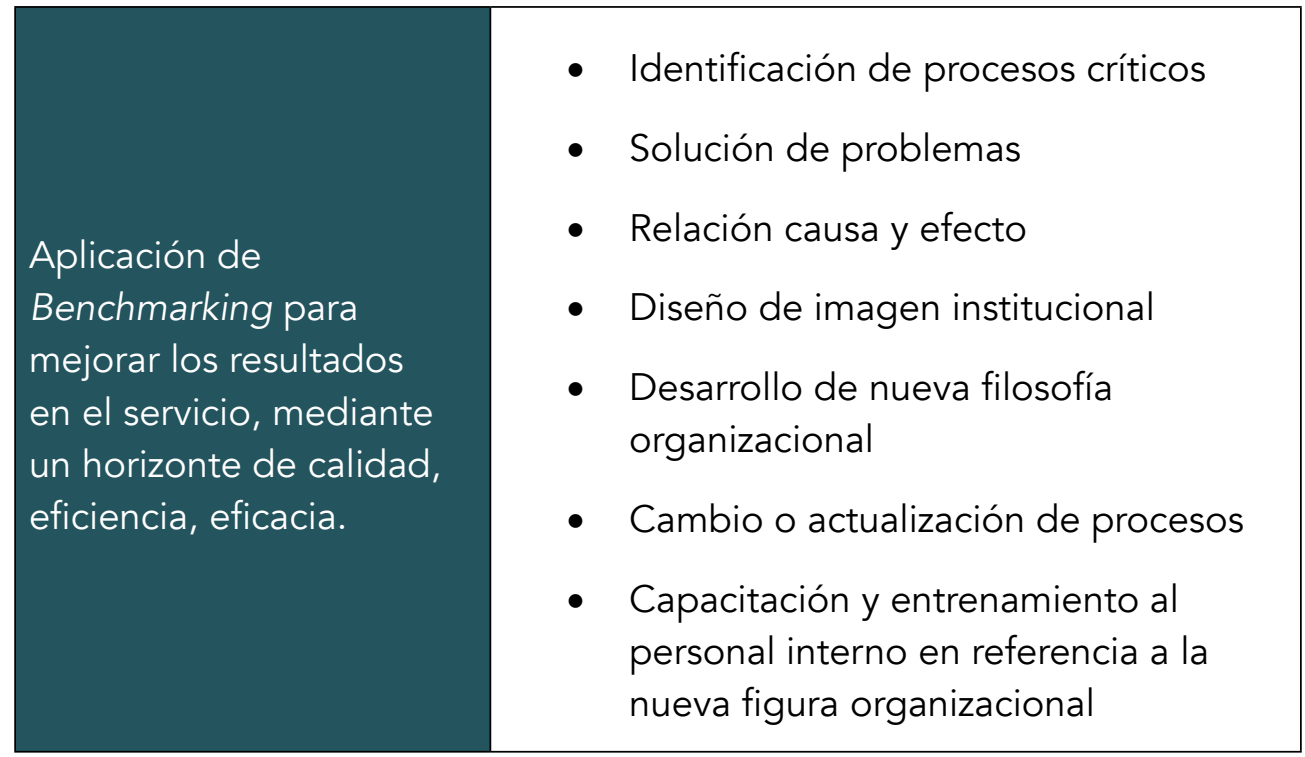

Elaborado por: Los investigadores. 
En cuanto al análisis de la aplicación del Benchmarking, se estableció que es necesario que se elaboren lineamientos concretos como herramienta gerencial práctica, que facilite la gestión y la toma de decisiones, para cubrir las expectativas de los usuarios significadas en lo que se reconoce como la satisfacción del cliente. Es decir, que los procesos vayan en conformidad con la efectividad institucional, para que puedan alcanzar ventaja competitiva a través del cuidado de los intangibles y de la imagen institucional.

Además, es necesario realizar diversas revisiones en los contextos de la planificación interna, para ofrecer soluciones de cambio en una proyección estratégica que sincronice efectivamente los procesos en los diferentes departamentos con el fin de disminuir la burocratización mecanizada y el papeleo innecesario. Si ponemos en claro que las instituciones públicas necesitan al cien por ciento la ejecución del Benchmarking se podría presentar un modelo de cambio como se ilustra en la siguiente tabla: 
Tabla 3: Cuadro comparativo sobre la aplicación de Benchmarking en atención a los usuarios

\section{Sin Benchmarking}

\section{Estrategias de atención a los usuarios}

Sin calidad en la atención al usuario.

Insatisfacción en los servicios.

Baja percepción y desconfianza del usuario.
Cultura de servicio al usuario y análisis de mercado.

Satisfacción en la atención y los servicios.

Altos niveles de confiabilidad y recomendación y fidelización.

\section{Aplicación de la innovación y desarrollo interno}

Carencia de foco interno y externo.

Sin planificación y visión estratégica.

Sin desafíos de innovación en los mercados cambiantes.
Activación del cambio en base a una visión estratégica.

Objetivos y estrategias con enfoque global (interno y externo).

Desarrolla tendencias y mejores prácticas de crecimiento interno.

\section{Implementación de medias de productividad}

Sin eficiencia ni rendimiento.

Desperdicio de tiempo y de recursos administrativos y financieros.

Poca evolución.

Bajo compromiso de los colaboradores.
Comprensión concreta de las prácticas de cambio institucional.

Solución de problemas reales con base en resultados de tiempo y costo.

Elección de nuevas ideas con el uso de tecnologías comprobadas.

Alto compromiso del personal interno

\section{Mejores prácticas internas de cambio estructural}

Falta de progreso institucional.

Reactividad al cambio.

Falta de proactividad.

Falta de capacidad de respuesta.
Ejecución de la calidad y del sistema de calidad basada en modelos.

Mejora continua en procesos.

Involucramiento del nuevo desarrollo institucional en todo el organigrama funcional.

Elaborado por: Los investigadores. 
Tomando como referencia el estudio de los autores Kinnell \& Garrod (1995) donde desarrollaron el Benchmarking como herramienta para el proceso de atención al cliente en bibliotecas de Inglaterra, se determinan los siguientes pasos para una buena atención: Identificación de factores clave de éxito, Diagrama de procesos y subprocesos, Identificación de procesos claves, Análisis de los resultados con diferenciación en el rendimiento y Equipamiento de mejores prácticas (Kinnell \& Garrod, 1995).

A continuación (figura 9), se representan algunos elementos que ayudarán a integrar la calidad en el servicio de atención al usuario en las instituciones públicas, por medio del Benchmarking.

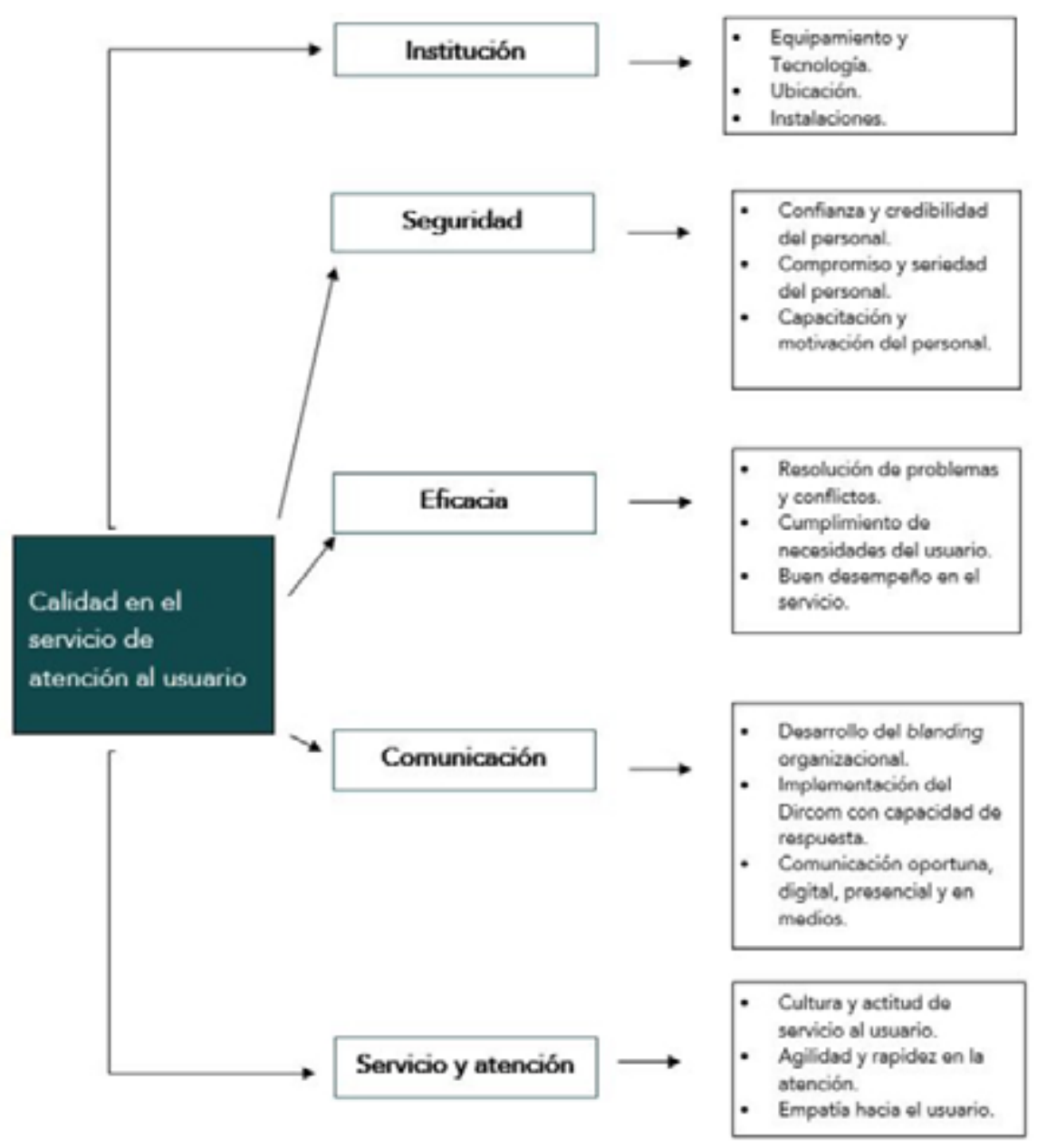

Figura 9: Representación de la calidad en el servicio de atención al usuario Fuente: Elaboración a partir de (Kinnell \& Garrod, 1995) y (Hernández Rodríguez, 2014). 
La herramienta del Benchmarking es un medio para conseguir un buen diagnóstico crítico del desempeño administrativo de cualquier institución. Es por esta razón que es una buena alternativa y una solución adecuada para que en las instituciones públicas lo fomenten; también, ayuda a la autoevaluación y a un análisis interno, porque considera lo que hace y debe hacer la alta dirección y el personal que se implica en la ejecución de los procesos internos. Se vuelve una posible alerta en el mejoramiento de los servicios.

Cabe mencionar que el Benchmarking no hace milagros y, más aún, en las instituciones del Estado que requieren cambios estructurales, que deben tener siempre, como meta, la satisfacción del usuario para alinear los procesos; es necesario que se rompan los esquemas de burocratización y falta de desarrollo, por medio de políticas, normas y leyes que sean dadas desde la Función Ejecutiva del Estado, para su buen funcionamiento.

Esencialmente todos, sin excepción, llegamos a ser sujetos de consumo, siendo el servicio de atención al usuario una variable que diariamente debe ser monitoreada por ejecutivos, directores, administradores, ministros, coordinadores, etc.

De todo lo expresado anteriormente se desprende las siguientes conclusiones:

Que las instituciones públicas poseen ineficiencia en los procesos y un trato desfavorable hacia los usuarios, ya que se evidencia la falta de cultura de servicio, tanto en el área de atención al usuario como en las relaciones interpersonales, dando presencia a un servicio escaso en calidad y calidez, evidenciando la existencia de la burocratización dentro de la trazabilidad de un trámite el cual causa tiempos muertos, largas esperas, reprogramaciones o reprocesos que influye en el costo- beneficio del usuario final, aumentando así las quejas y la insatisfacción.

Además, se puede mencionar que la falta de desarrollo organizacional e imagen corporativa en este tipo de instituciones se debe a burocracia, a la estructura institucional y la falta de motivación del personal porque la comunicación es lenta, afectando a la estructura inicial de la cadena de trazabilidad, la cual, influye profundamente en el rendimiento de las operaciones diarias, porque para tener resultados es necesarios que un proceso pase por muchas instancias. 
Por último, la constante presencia de la burocratización mecanizada en las instituciones públicas, está ligado también a la falta de capacitación del personal interno, a la inexistencia de manuales de atención al usuario o de procesos automatizados que recolectan la información de manera digital pero que así tienen que ser validado de forma física por un funcionario, lo que genera el deterioro en la calidad de atención al usuario o la falta de información. 


\section{Referencias}

Bellidov, F. J. (2017). La burocratización del poder político: Notas sobre sus consecuencias en las democracias parlamentarias. Astrolabio. Revista Internacional de Filosofía, 1-7.

Bozeman, B. (2015). Causas, efectos y eficacia de la burocratización en las administraciones públicas nacionales: desarrollando reformas sensibles a culturas políticas singulares. Revista del CLAD Reforma y Democracia, $N^{\circ}$. 63, 5-32.

Campos, N. F., \& Nugent, J. B. (1999). Development Performance and the Institutions of Governance: Evidence from East Asia and Latin America, en World. Development, Vol. 27 N³, 439-452.

Dávila Espinosa, M. G. (2015). Influencia del sistema burocrático en la satisfacción del personal de ventanilla del SRI. Quito.

Eisenstadt, S. N. (1959). Bureaucracy, Bureaucratization, and Debureaucratization en Administrative Science. Quarterly, Vol. 4 N³, 302-320.

Gutiérrez Cadena, G. (2019). La calidad del servicio y la satisfacción de los clientes. [Tesis maestría, Universidad Espíritu Santo]. Repositorio Insitucional.http://repositorio.ucsg.edu.ec/handle/3317/73/ browse?type $=$ subject\&order $=$ ASC\&rpp $=85 \&$ value $=S E R V I C I O+A L+-$ CLIENTE.

G. Schiffman, L., \& Lazar Kanuk, L. (2011). Comportamiento del consumidor (Décima ed ed.). México DF.: Pearson.

Gómez Cárdenas, R. (2016). Modelo ServQual EPMAPA. https://d1wqtxts1xzle7.cloudfront.net/44529958/Modelo_SERVQUAL_-_EPMAPA-SD. pdf?1460102121=\&response-content-disposition=inline\%3B+filename\%3DLa_Calidad_en_los_Servicios_Publicos_des.pdf\&Expires $=1591503805 \&$ Signature $=$ ao2f6 FT2irM7qMtvCxEOshUXZy VzTbpT8HhcOB

Goodsell, C. T. (2003). The Case for Bureaucracy: a Public Administration Polemic,. Thousand Oaks Sage.

Gormley, W. T., \& Balla, S. J. (2012). Bureaucracy and Democracy: Accountability and Performance. Thousand Oaks, Sage. 
Hernández Rodríguez , C. (2014). El servicio al cliente como factor de competitividad y calidad en las empresas: Caso de las Organizaciones escolares particulares. Revista Ciencia Administrativa del Instituto de Investigaciones y de Estudios Superiores, 10-20.

Hernández Sampieri., R., \& Mendoza torres, C. P. (2018). Metodología de Investigación (7ma ed.). México: Mc Graw Hill.

INEC. (2015). Calidad de los servicios en Ecuador. Talleres Gráficos del Inec. https://www.ecuadorencifras.gob.ec/el-inec-mide-la-calidad-de-losservicios-publicos/.

Kinnell, M., \& Garrod, P. (1995). Benchmarking and its relevance to the Library and information. British Library. Research and Development Department Project. Northumberland: s.n.

Mendoza Aquino, J. A. (2009). Medición de la calidad de servicios. (El Cid Editor I apuntes, Editor) Ebrary: http://site.ebrary.com/lib/ bibliovirtualuidesp/detail.action?docID=10316508\&p00=mendoza+ aquino

Nef, J. (2007). Public Administration and Public Sector Reform in Latin America. The SAGE Handbook of Public, 32-48.

Pérez Leal, J. (2018). Qué es la matriz de vester para la priorización de problemas? Blogasesoria de tesis y trabajos de grado: https://asesoriatesis1960.blogspot.com/2018/07/que-es-la-matriz-de-vester-parala.html

Pérez , V. (2014). Calidad en la atención al cliente. Pautas para garantizar la excelencia en el servicio. Ideas Propias.

Rodríguez, C. (1987). Administración Pública Ecuatoriana. Quito: ILDIS.

Torres Gordillo, J. J., \& Perera Rodriguez, V. H. (2009). Cálculo de la fiabilidad y concordancia entre codificadores de un sistema de categorías para el estudio de un foro online en e-learnig. Revista de Investigación Educativa, Vol. 27, N 1, 89-103.

Vélez Rendon, J. C. (2012). Modernización del Estado y administración pública: entre la burocratización y la tecnocracia. Instituto de Estudios Políticos, 75-95.

Zapata Mora, B. (2018). Atención al cliente con falencias en Ecuador. https:// www.eluniverso.com/noticias/2018/04/26/nota/6731814/atencion-cliente-falencias-ecuador 